\title{
24GHz CMOS Power Amplifier for Automotive Radar
}

\author{
Myeong-U Sung ${ }^{1}$, Geun-Ho Choi ${ }^{1}$, Habib Rastegar ${ }^{1}$, Shin-Gon $\mathrm{Kim}^{1}$, Murod \\ Kurbanov ${ }^{1}$, Pushpa Chandrasekar ${ }^{1}$, Jae-Hwan Lim ${ }^{2}$, Seong-Jin Heo ${ }^{1}$, Jee-Youl \\ $\mathrm{Ryu}^{1 *}$, Seok-Ho Noh ${ }^{3}$ and Min Yoon ${ }^{4}$ \\ ${ }^{1}$ DeparAtment of Information and Communications Engineering, Pukyong \\ National University \\ ${ }^{2}$ Busan Regional Industry Evaluation Agency, Dongnam Institute for Regional \\ Program Evaluation \\ ${ }^{3}$ Department of Electronic Engineering, Andong National University, Busan \\ ${ }^{4}$ Department of Statistics, Pukyong National University \\ Corresponding author (Email: ryujy@pknu.ac.kr)
}

\begin{abstract}
This paper proposes $24 \mathrm{GHz}$ CMOS power amplifier (PA) for the short-range automotive collision avoidance radar. The proposed amplifier is implemented using TSMC 0.13um RF CMOS technology with $f_{T} / f_{\max }$ of $120 / 140 \mathrm{GHz}$. It is also powered by a $1.5 \mathrm{~V}$ supply. The layout optimization technique is used to reduce total die size and parasitic capacitances. To improve power gain of the amplifier, the circuit has a 2-stage architecture and cascode scheme in the first stage. The proposed circuit showed the smallest chip size of $0.25 \mathrm{~mm}^{2}$, the highest power gain of $29.3 \mathrm{~dB}$, the lowest power dissipation of $42 \mathrm{~mW}$, and the maximum PAE of $18.5 \%$ as compared to recently reported research results.
\end{abstract}

Keywords: $24 \mathrm{GHz}, \mathrm{CMOS}$ power amplifier, short range automotive collision avoidance radar

\section{Introduction}

The increasing demand for low-power and low-cost radio frequency integrated circuits (RF ICs) in millimeter-wave applications requires high-level integration of RF front-end, analog blocks and digital blocks for system-on-a-chip (SoC). The rapid growth in wireless communications has also resulted in a strong motivation toward developing GHz-band high performance RF systems [1]-[11]. The design and implementation of single-chip transceivers has already been demonstrated in CMOS technologies for RF CMOS ICs. These systems have lots of applications such as various portable products, automotive collision avoidance radars, wireless local networks, etc. The unlicensed industrial, scientific, and medical (ISM) frequency band at $24 \mathrm{GHz}$ is attractive for commercial, industrial and automotive applications [1]-[8]. For wireless communication circuits, a power amplifier is one of the transceiver's key elements. The $24 \mathrm{GHz}$ automotive applications using the standard CMOS technology pose a particular challenge on circuit design because of the high robustness and performance. Numerous publications have reported $24 \mathrm{GHz}$ radar in CMOS technology, and active study on $24 \mathrm{GHz}$ radar is still underway [1]-[8], [12][14].

In this paper, we present a small-area, low power, and high-gain $24 \mathrm{GHz}$ power amplifiers (PA) for the short-range automotive radar. This circuit is fabricated using TSMC $0.13 \mu \mathrm{m}$ RF CMOS process, and it is powered by a $1.5 \mathrm{~V}$ supply. The proposed circuit is designed using a 2 -stage architecture and cascode scheme in the first stage. We 
used the layout optimization technique for $24 \mathrm{GHz}$ band to reduce parasitic capacitances.

\section{Design and Analysis of Power Amplifier}

\subsection{Overview of $24 \mathrm{GHz}$ Radar}

The radar-based autonomous cruise control (ACC) at $77 \mathrm{GHz}$, first introduced from Mercedes-Benz in 1999, is widely available in many high and mid class automotive vehicles. Silicon-based $24 \mathrm{GHz}$ short-range automotive radars have been investigated by both industry and academia in the last 20 years [5]-[6], [15]. Most of all, automotive collision avoidance radar using $24 \mathrm{GHz}$ band offers safety functions such as pre-crash sensing and collision. Most well known car companies and supplies are already working on the development of the next generation vehicle known as Advanced Safety Vehicle (ASV). Therefore, next generation radars may well be required to support $24 \mathrm{GHz}$ band for compatibility and lower overall cost [5]-[9], [15].

The short-range radar (SRR) sensors with coverage up to 30 meters are under development for various further applications. The SRR will be used to expand ACC to stop-and-go operation, to perform pre-crash warning, to act as parking aid, or to warn during backing up or changing lanes [15]. The short range radar sensors can enable a variety of applications such as ACC support with stop and go functionality, collision warning collision mitigation, blind spot monitoring, forward and reverse parking aid, lane change assistant, rear crash collision warning, etc. as described in Figure 1 [15].

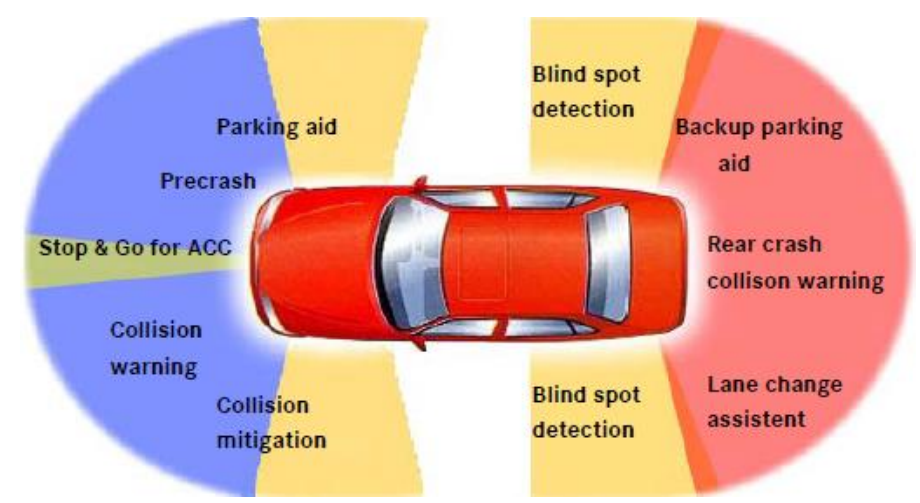

Figure 1. Possible Application for SRR

\subsection{Design of 24GHz CMOS Power Amplifier}

\section{(1) Design and Analysis}

The 24GHz CMOS PA is implemented using TSMC 0.13 $\mu$ m RF CMOS technology. The unity current gain cut-off frequency $\left(f_{T}\right)$ and the maximum oscillation frequency $\left(f_{\max }\right)$ have $120 \mathrm{GHz}$ and $140 \mathrm{GHz}$, respectively. Successful integration of the PA at $24 \mathrm{GHz}$ depends on minimization of parasitic capacitances and losses to maintain adequate gain, designing with low voltage swings for low breakdown devices, and achieving sufficient linearity required for spectrally efficient and variable envelope modulation scheme [12][14]. Multi-stage PA topologies are essential to obtain high gain at W-band, and it is really true in the case of CMOS technology [12]. It is very important to get high gain, since nanoscale MOSFET is degraded by $3 \mathrm{~dB}$ due to the limited load impedance that can be realized at the drain output at the high frequency [12].

Optimized integration of the PA at $24 \mathrm{GHz}$ depends on minimization of parasitic capacitances and losses to maintain adequate gain, designing with low voltage swings for 
low breakdown devices, and achieving sufficient linearity required for spectrally efficient and variable envelope modulation scheme [12].

Multi-stage PA topologies are essential to obtain high gain at W-band, and this is really true in the case of CMOS technology. It is very important to get high gain, since nanoscale MOSFET is degraded by $3 \sim 4 \mathrm{~dB}$ due to the limited load impedance that can be realized at the drain output at the high frequency and the losses in the matching networks [12]. The optimal distribution of power gain and bias current (and hence $P_{1 d B}$ ) for the inter-stage of the PA can be obtained using the well-known expression for linearity in a cascaded system as shown in Equation (1).

$$
\frac{1}{O P_{1 d B_{-} \text {cascade }}}=\frac{1}{O P_{1 d B_{-} 3}}+\frac{1}{O P_{1 d B_{-} 2} G_{3}}+\frac{1}{O P_{1 d B_{-} 1} G_{2} G_{3}}
$$

Figure 2 shows the proposed $24 \mathrm{GHz}$ CMOS PA. The PA consists of commonsource stage with inter-stage conjugate matching operating in Class-A mode, and it is powered by a $1.5 \mathrm{~V}$ supply. This circuit also has 2 -stage cascade structure to achieve higher gain due to the larger output impedance and the alleviated miller capacitance. To input impedance matching, we use inductor $L_{l}$ and MIM capacitor, $C_{l}$. The inter-stage matching networks are designed using $L_{2} \sim L_{3}$ and $C_{2}$. The $L_{4}$ and $C_{3}$ are used for output matching network. To reduce RF noise, power supply noise and EMI, we used a decoupling capacitor, $C_{4}$. We also realized an optimization technique in width and length of transistors, $M_{1} \sim M_{3}$.

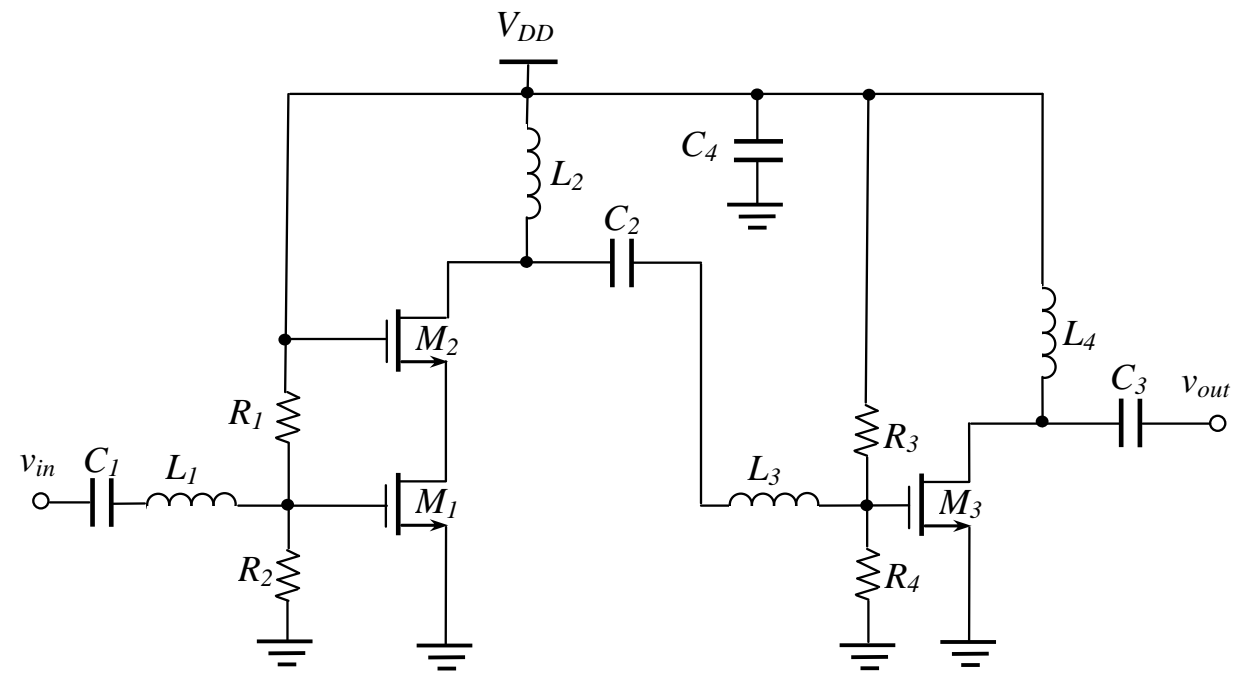

Figure 2. 24GHz CMOS Power Amplifier

\section{(2) Measurement Set-up}

Figure 3 shows S-parameter network analyzer measurements for PA Vector network analyzers measure the transmission and reflection characteristics of devices and networks by applying a known swept signal from a synthesized source. Device reflection parameters such as input and output reflection coefficients, return loss, complex impedance, and transmission parameters such as reverse isolation and voltage gain can be measured using the instrumentation. Consider the test set-up for S-parameter measurement shown in Figure 3. It contains vector network analyzer, wafer probe station, DC power supply, digital multi-meter and PC. The wafer probe station has two RF probes to provide RF input powers at ports 1 and 2 of PA, and two DC probes to provide DC power and ground. The measurements have been used here represent 2-port 
measurements. These measured values are translated into PA specifications such as input impedance, voltage gain, reflection coefficients and reverse isolation.

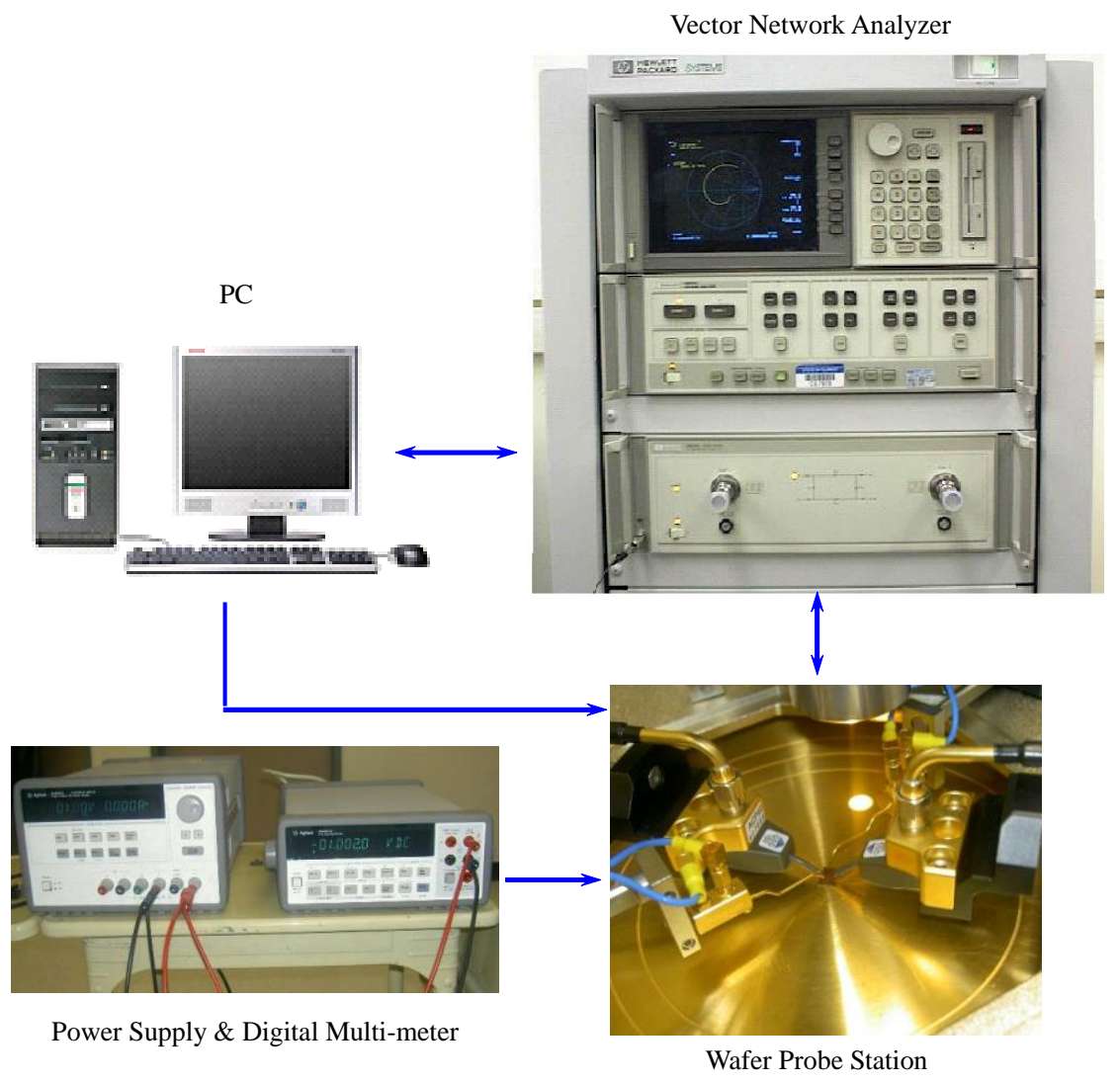

Figure 3. S-Parameter Measurement Set-Up

Figure 4 shows the measurement set-up for the S-parameter of the PA. Vector network analyzer and probe station measure the S-parameter by applying a known swept signal from a synthesized source. The S-parameter measurement has been used here which represent 2-port measurements. The powers of $-20 \mathrm{dBm}$ are applied from the synthesized sources at both port 1 and port 2 . We applied the attentions of $0 \mathrm{~dB}$ at both port 1 and port 2. The measured S-parameter was transferred to voltage gain, return losses and reverse isolation using conventional equations of ADS or MATLAB tool.

Let's consider Figure 4 with the source $\left(V_{i n}\right)$ forming part of a network analyzer with a matched load $\left(Z_{L}=50 \Omega\right)$ at port 2 to measure transfer function $\left(S_{21}\right)$ for the PA. The $S_{21}$ can be obtained by applying an incident wave at port $1, V_{I}^{+}$, and by measuring the outcoming wave at port $2, V_{2}^{-}$. This is equivalent to the transmission coefficient from port 1 to port 2. Since $S_{21}$ is a measurement of the gain at the network analyzer output, the transfer function $H(f)$ can be derived to be reference [2].

$$
H(f)=S_{21}=\left.\frac{V_{2}^{-}}{V_{1}^{+}}\right|_{V_{2}^{+}=0} .
$$

Figure 5 shows simplified high-frequency small-signal model for the input side of the PA. The simultaneous matching including the effect of the load impedance is considered for the simplified small-signal MOS models and inductor model with lossy resistances. The transistor is replaced with hybrid- $\pi$ model, and the inductor is replaced with series lossy resistance $R_{L}$ and its inductance $L$. From hand analysis of the PA, the input impedance, power gain, S11/S12/S22 and transfer functions are obtained. 


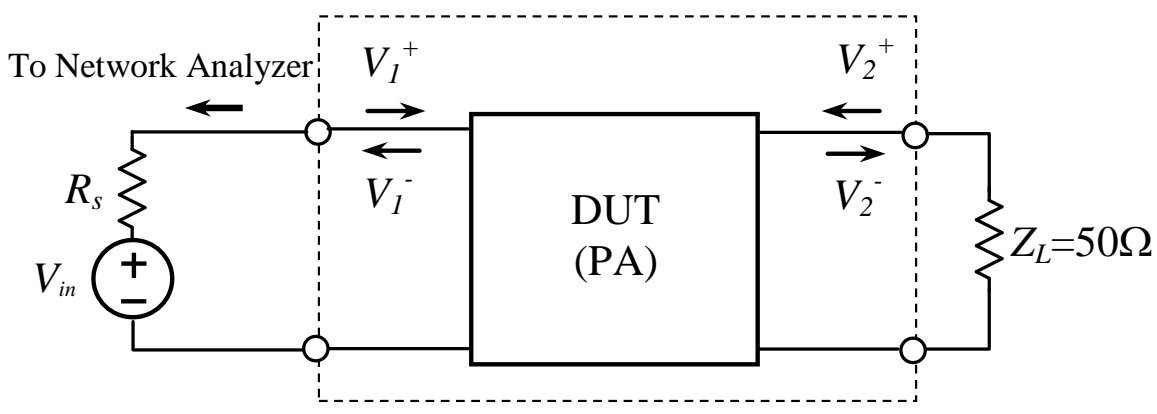

Figure 4. 2-Port Measurement Set-Up

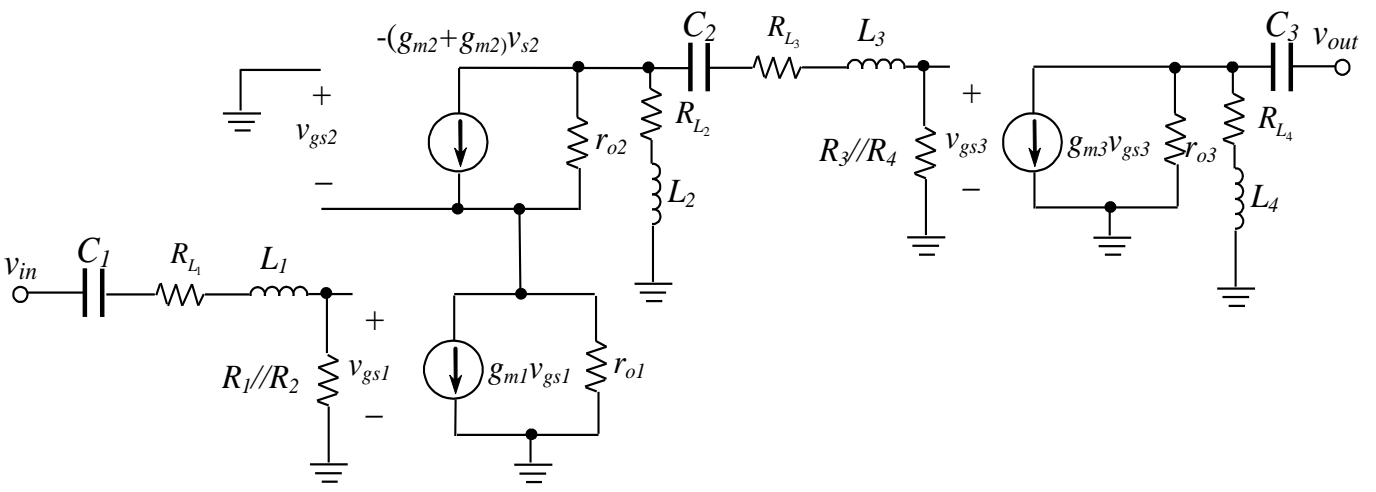

Figure 5. High-Frequency Small-Signal Model for the PA

\section{Results}

The extracted S-parameter models have been included in circuit simulation using Agilent advanced design system (ADS). The circuit is simulated using Agilent ADS, and full-wave EM analysis is performed for all the passive structures. The speed of the bias mechanism is determined by the bias resistor and gate-source parasitic capacitor at the gate of the lower FET, and the settling time is about $4 \mathrm{~ns}$ based on the time-domain simulation when the input power of the PA changes from 5 to $-5 \mathrm{dBm}$. The amplifier is tested with a power supply of $1.5 \mathrm{~V}$, and the DC current drawn without applied RF input signal is $28 \mathrm{~mA}$.

The input and output pads are laid out in ground-signal-ground (GSG) configuration, with a pitch of $150 \mu \mathrm{m}$ to do wafer level testing for the PA using a probe station with network analyzer. The measurements for the PA used here 2-port measurements. The measurements are based on a separate PA test chip. The powers of $-20 \mathrm{dBm}$ are applied from the synthesized sources at both port 1 and port 2 . We applied the attenuators of $0 \mathrm{~dB}$ at both port 1 and port 2. The measured S-parameter was translated into power gain, reflection coefficients (S11/S22), reverse isolation (S12), and input impedance.

The PA is tested by probing the input and output ports. The input, output and power supply pads are laid out in ground-power-ground (GPG) and GSG configurations with a pitch of $50 \mu \mathrm{m}$ to perform packaged level testing. The power and ground pads are wirebonded to the testing board.

Figure 6 shows the S-parameter (S11, S12, S21 and S22) of the PA at the frequency range of 21.5 to $27.5 \mathrm{GHz}$. The PA achieves an excellent peak voltage gain (S21) of approximately $26 \mathrm{~dB}$ at $24 \mathrm{GHz}$, and the $\mathrm{S} 12$ indicates that the reverse isolation from the output to the input is also excellent value of approximately $-39 \mathrm{~dB}$ at $24 \mathrm{GHz}$. 
The input reflection (S11) and output reflection (S22) indicate how well the input and output are matched to $50 \Omega$, respectively. The S11 showed excellent value of $-24.5 \mathrm{~dB}$ at $24 \mathrm{GHz}$, but it was not designed for an input match to $50 \Omega$ for the entire $21 \sim 28 \mathrm{GHz}$ band. It should be noted that the requirement on S11 could be relaxed if the amplifier is integrated with the transmitter stages. Both $\mathrm{S} 11$ and $\mathrm{S} 22$ are $<-5 \mathrm{~dB}$ in the range of $21 \sim 28 \mathrm{GHz}$.

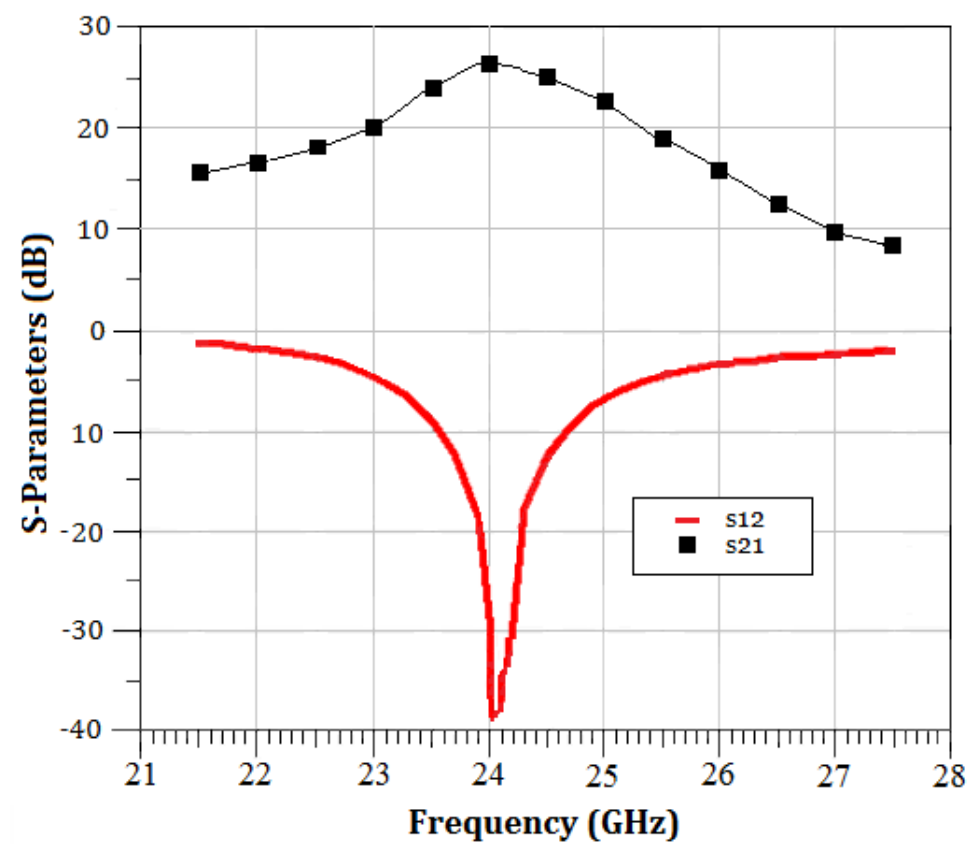

(a) Reverse isolation and voltage gain

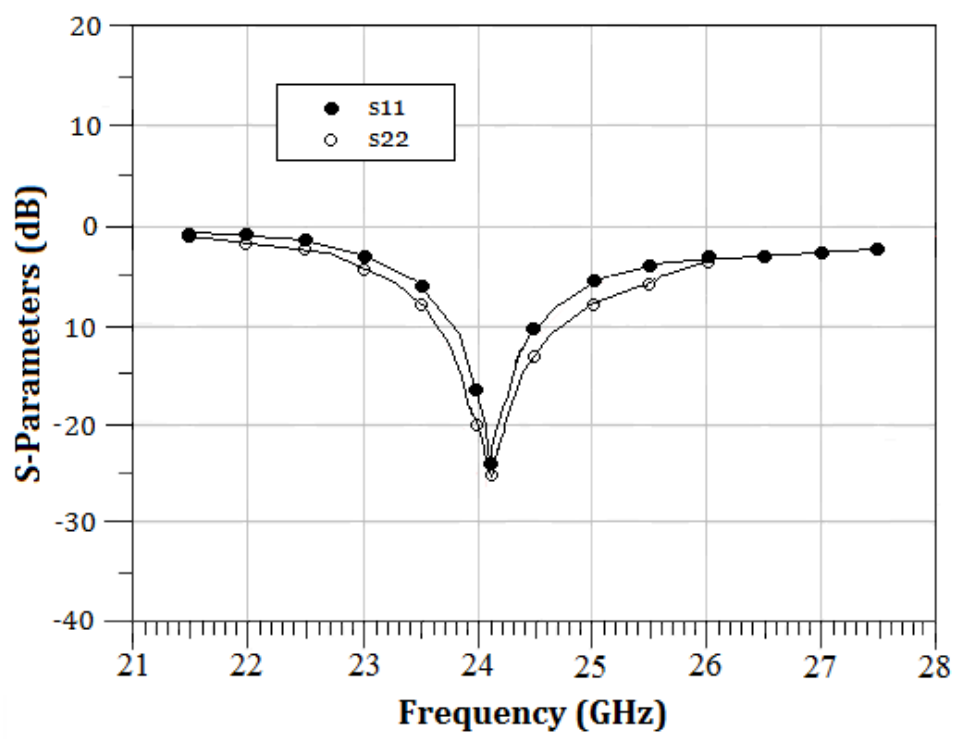

(b) Input and output reflection coefficients

Figure 6. S-parameters for the PA

Figure 7 shows the large signal performance of the PA, where an output 1-dB compression point $\left(\mathrm{OP}_{1 \mathrm{~dB}}\right)$ of $18 \mathrm{dBm}$ and a saturated output power of $22.3 \mathrm{~dB}$ were obtained at $24 \mathrm{GHz}$ with a $1.5 \mathrm{~V}$ supply. The simulated output power agrees well with hand analysis, which predicts an output $\mathrm{P}_{1 \mathrm{~dB}}$ of $-12.9 \mathrm{dBm}$. The linearity and gain depends on the bias current of the final stage. Since the PA has available dynamic range, output 
power and power gain are changed for the input power. As seen in Figure 7, the PA showed the highest power gain of $29.3 \mathrm{~dB}$, and high output 1-dB compression point $\left(\mathrm{OP}_{1 \mathrm{~dB}}\right)$ of $18 \mathrm{dBm}$ at the input $1-\mathrm{dB}$ compression point $\left(\mathrm{IP}_{1 \mathrm{~dB}}\right)$ of $-12.9 \mathrm{dBm}$, respectively. These results verify that the proposed amplifier shows excellent output power and power gain as compared to recently reported results [12]-[14], [16].

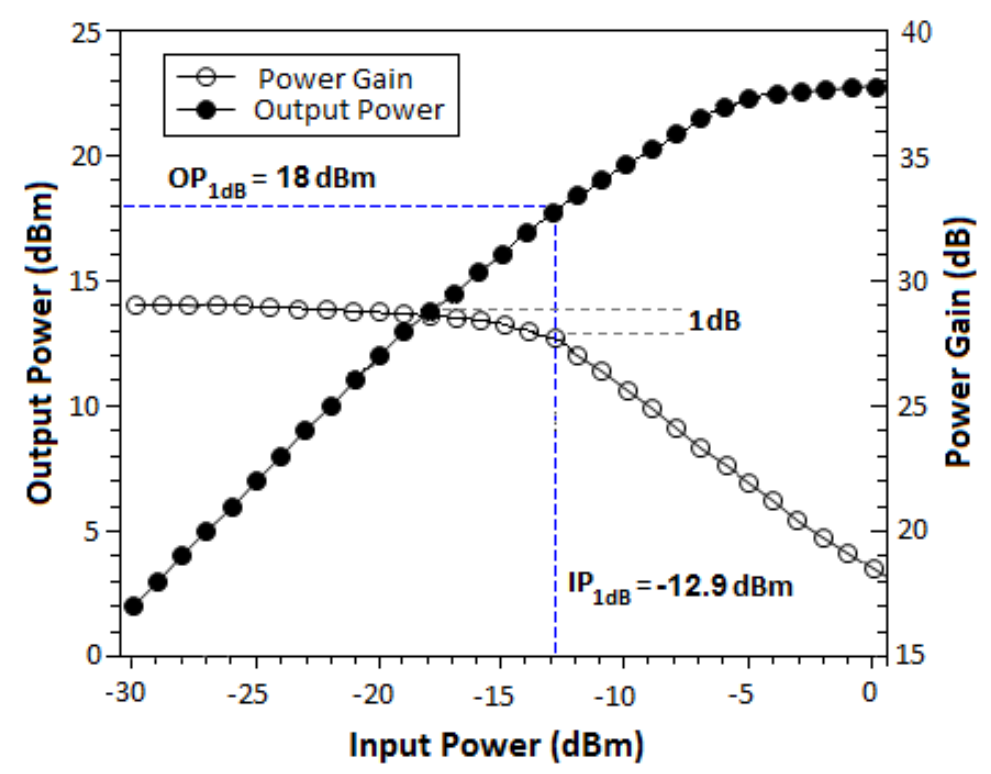

Figure 8. Output Power and Power Gain versus Input Power

Figure 9 shows output power and power-added efficiency (PAE) versus input power at the frequency of $24 \mathrm{GHz}$. The results showed the highest power gain $\left(\mathrm{P}_{\mathrm{sat}}\right)$ of $22.3 \mathrm{~dB}$ and the highest maximum PAE $\left(\mathrm{PAE}_{\max }\right)$ of $18.5 \%$ as recently reported research [12]-[14], [16]. The proposed PA for automotive radar applications is required to be good phase linearity with small group delay variations to sustain good shaped pulse forms using transmission antenna. The high power gain and high $\mathrm{PAE}_{\max }$ verify that the proposed PA shows excellent phase linearity.

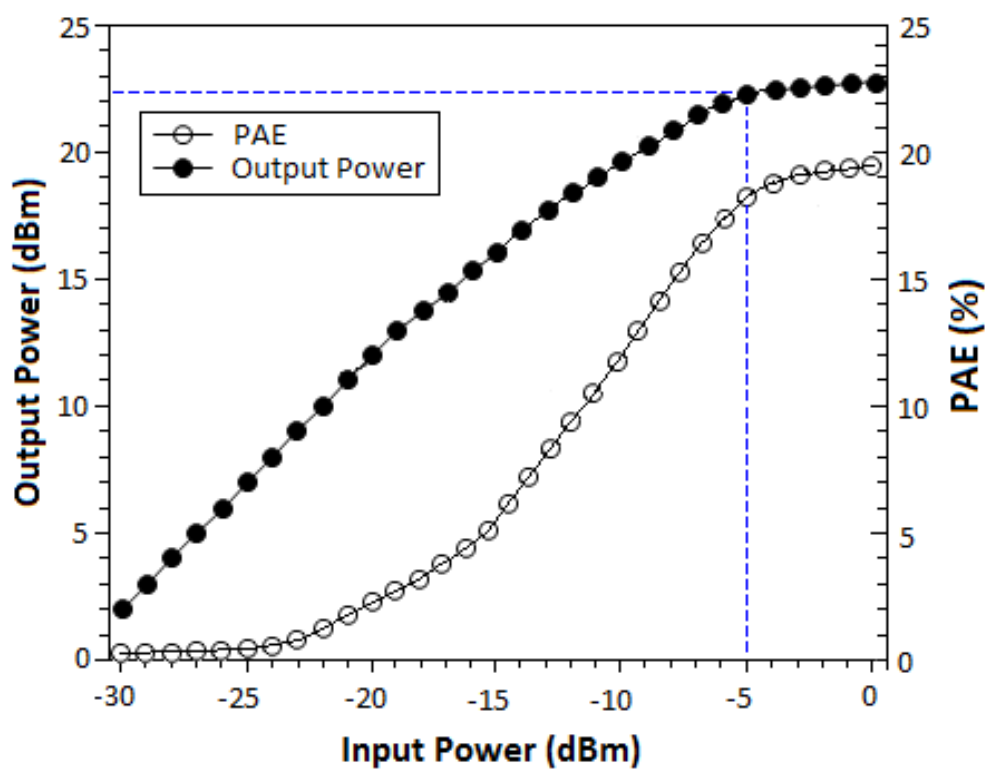

Figure 9. Output Power and PAE versus Input Power 
Figure 10 shows power-added efficiency (PAE) and saturated output power $\left(\mathrm{P}_{\mathrm{sat}}\right)$ versus input power when the amplifier is operating under saturated output power condition driven by the $-5 \mathrm{dBm}$ input. Maximum output power of $20.3 \mathrm{dBm}(107.2 \mathrm{~mW})$ is achieved at $24 \mathrm{GHz}$. At $24 \mathrm{GHz}$, the final gain stage of the power amplifier achieves a maximum output power density (power per source area) of $2 \mathrm{~mW} / \mu \mathrm{m}^{2}$. The PAE exceeds $6 \%$ between 20 and $29 \mathrm{GHz}$. The amplifier has power gain of more than $20 \mathrm{~dB}$ from 20 to $29 \mathrm{GHz}$ with $3 \mathrm{~dB}$ gain flatness. Over $15 \mathrm{~dB}$ gain at maximum output power level facilitates integration of the amplifier in a transceiver as fewer pre-driver stages are required. When biased at $14 \mathrm{~mA}$ (i.e., $50 \%$ of $28 \mathrm{~mA} \mathrm{DC}$ current with no RF signal applied), there is less than $3 \mathrm{~dB}$ reduction in output power and slightly power PAE, despite the transistor transconductance $g_{m}$ being reduced by half for all three stages. As shown in Figure 10, the proposed PA showed a high-saturated output power of $20.3 \mathrm{dBm}$ and maximum PAE of $18.5 \%$ at the operation frequency of $24 \mathrm{GHz}$, respectively.

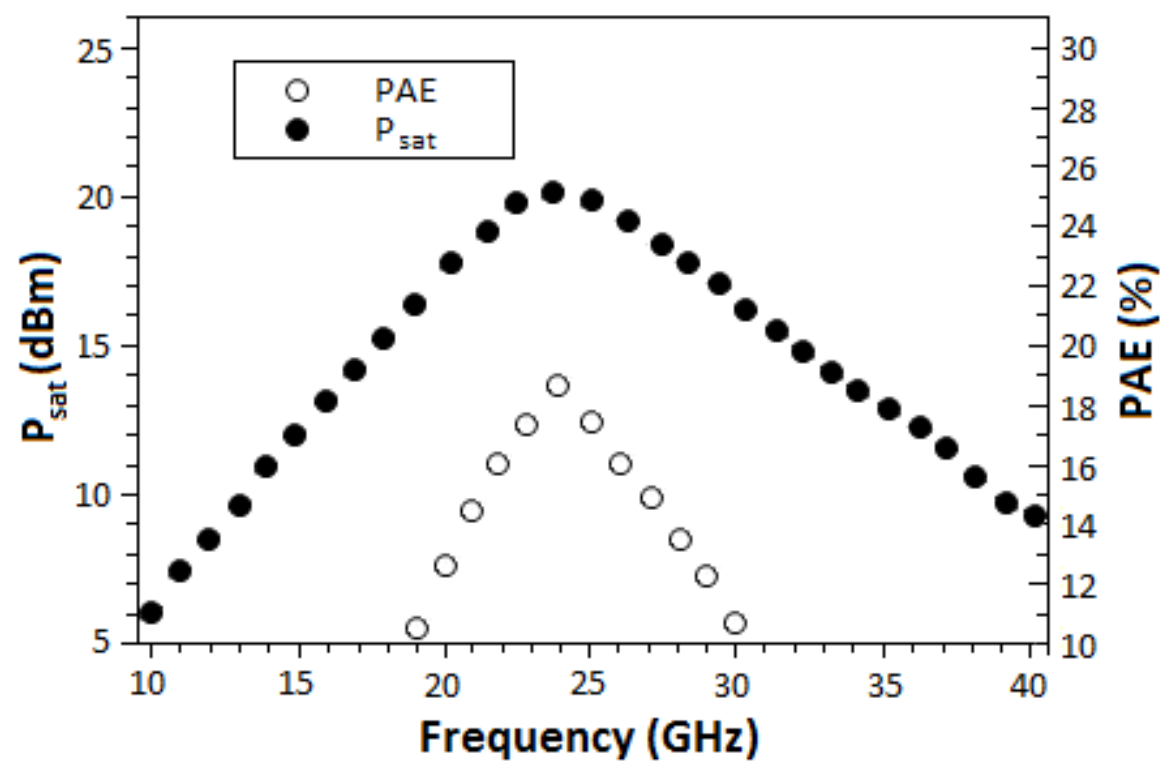

Figure 10. Saturated Power $\left(P_{\text {sat }}\right)$ and PAE versus Frequency

The complete measured performance comparison of the PA is summarized in Table 1. The circuit correlation function is determined by varying the delay $R_{x}$ trigger in the pulse generator. Due to the circuit mask constraints at $24 \mathrm{GHz}$, multiple pulses need to be integrated to raise the signal above the noise floor. This is demonstrated in measurement results of Table 1, which shows the integrator output after coherent integration of 100 pulses for each delay setting. A $1 \mathrm{~ns}$ pulse is generated, corresponding to a $12 \mathrm{~cm}$ range resolution, and the delay is varied in 100ps steps, corresponding to $2 \mathrm{~cm}$ range accuracy. As can be seen from this comparison, the proposed CMOS PA showed the highest power gain of $29.3 \mathrm{~dB}$, the lowest power dissipation of $42 \mathrm{~mW}$, the smallest chip size of $0.25 \mathrm{~mm}^{2}$, the highest saturated power $\left(\mathrm{P}_{\text {sat }}\right)$ of $20.3 \mathrm{dBm}$, and the maximum power-added efficiency (PAE) of $18.5 \%$ as compared to recently reported research results. This PA also showed very high output IP3 of $18 \mathrm{dBm}$, very low input/output reflection coefficients (S11/S22) of $-24.5 /-25.1 \mathrm{~dB}$ and very low reverse isolation (S12) of $-41.2 \mathrm{~dB}$.

\section{Conclusions}

In this paper, we presented small-area low-power and high gain $24 \mathrm{GHz} \mathrm{CMOS}$ power amplifier based on automotive collision avoidance radar application. The proposed circuit was fabricated using TSMC $0.13 \mu \mathrm{m}$ RF CMOS technology. The layout techniques for RF circuits were used to reduce parasitic capacitances at the 
frequency range of $22 \sim 26 \mathrm{GHz}$. The proposed circuit showed the highest power gain of $29.3 \mathrm{~dB}$, the lowest power dissipation of $42 \mathrm{~mW}$, the smallest chip size of $0.25 \mathrm{~mm}^{2}$, the highest saturated power of $20.3 \mathrm{dBm}$, and the maximum power-added efficiency (PAE) of $18.5 \%$ as compared to recently reported research results. This circuit also showed very high output IP3 (OIP3) of 18dBm, very low S11/S22 of -24.5/-25.1dB and very low S12 of $-39.2 \mathrm{~dB}$.

Table 1. Comparison to Recently Reported 24GHz Power Amplifiers

\begin{tabular}{c||c|c|c|c|c}
\hline \multicolumn{1}{c||}{ References } & {$[12]$} & {$[13]$} & {$[14]$} & {$[16]$} & This Work \\
\hline \hline Parameters & 0.18 & 0.18 & 0.18 & 0.20 & 0.13 \\
\hline Process $(\mu \mathrm{m})$ & 24 & 22 & 24 & 24 & 24 \\
\hline Frequency $(\mathrm{GHz})$ & 7 & 15.4 & 14 & 23 & 18 \\
\hline OIP3 $(\mathrm{dBm})$ & 22.8 & 11.9 & 7 & 19 & $\mathbf{2 9 . 3}$ \\
\hline Power Gain $(\mathrm{dB})$ & 15.9 & 17.4 & 14.5 & 15 & $\mathbf{2 0 . 3}$ \\
\hline $\mathrm{P}_{\text {sat }}(\mathrm{dBm})$ & 163.8 & 108 & 280 & 939 & $\mathbf{4 2}$ \\
\hline DC Power Consumption $(\mathrm{mW})$ & $-14.3 /-17$ & $-8 /-10$ & $-6.9 /-16$ & $-12.4 / \mathrm{NA}$ & $\mathbf{- 2 4 . 5 / - 2 5 . 1}$ \\
\hline S11/S22 $(\mathrm{dB})$ & -64 & NA & -40 & -10.5 & $\mathbf{- 3 9 . 2}$ \\
\hline S12 $(\mathrm{dB})$ & 14.6 & 12 & 6.5 & 13 & $\mathbf{1 8 . 5}$ \\
\hline Maximum PAE $(\%)$ & 0.84 & 0.4 & 1.26 & 6.003 & $\mathbf{0 . 2 5}$ \\
\hline Chip Area $\left(\mathrm{mm}^{2}\right)$ & & & & & \\
\hline
\end{tabular}

\section{Acknowledgments}

This work was supported by the Regional Special Industry Cultivation (R\&D) Program (R0005056, Development of Small Portable Hardness Tester) funded by the Korea Institute for Advancement of Technology(KAIT, Korea), and this research was supported by Basic Science Research Program through the National Research Foundation of Korea (NRF) funded by the Ministry of Education (2015R1D1A3A01015753).

\section{References}

[1] S.-H. Noh, M.-U Sung, G.-H. Choi, H. Rastegar, S.-G. Kim, M. Kurbanov, P. Chandrasekar, S.-K. Choi, G.-M. Heo, K.-P. Kil, J.-Y. Ryu and M. Yoon, "Power Amplifier for $24 \mathrm{GHz}$ Radar Sensor", Proceedings of the 2016 International Conference on Future Information \& Communication Engineering, vol. 8, no. 1, DaNang, Vietnam, (2016).

[2] S.-H. Noh and J.-Y. Ryu, "Design of 24-GHz Power Amplifier for Automotive Collision Avoidance Radars", Journal of the Korea Institute of Information and Communication Engineering, vol. 20, no. 1, (2016), pp. 117-222.

[3] J.-Y. Ryu, "High-Gain Low-Area Power Amplifier for 77-GHz Automotive Radars", International Journal of Applied Engineering Research, vol. 11, no. 2, (2016), pp. 934-937.

[4] J.-Y. Ryu, "Low-Power Power Amplifier for 77-GHz Automotive Radars", International Journal of Applied Engineering Research, vol. 11, no. 1, (2016), pp. 661-663.

[5] S.-G. Kim, H. Rastegar, M. Yoon, C.-W. Park, K.-Y. Park, S.-K. Joung and J.-Y. Ryu, "High-Gain and Low-Power Power Amplifier for 24-GHz Automotive Radars", International Journal of Smart Home, vol. 9, no. 2, (2015), pp. 27-34.

[6] S.-H. Noh and J.-Y. Ryu, "Design of 24GHz CMOS Mixer for Automotive Collision Avoidance Radar", Journal of Korean Institute of Information Technology, vol. 12, no. 11, (2014), pp. 31-37.

[7] S.-H. Noh and J.-Y. Ryu, "Design of $24 \mathrm{GHz}$ Low Noise Amplifier for Short Range Radar of Automotive Collision Avoidance", Journal of Korean Institute of Information Technology, vol. 11, no. 10, (2013), pp. 23-28. 
[8] J.-H. Lee, and J.-Y. Ryu, S.-W. Kim, and S.-H. Noh, "Design of Radio Frequency Front-End for 77GHz Automotive Collision Avoidance Radar", Journal of Korean Institute of Information Technology, vol. 10, no. 7, (2012), pp. 13-19.

[9] J.-Y. Ryu and S.-H. Noh, "On-Chip Design-for-Testability Circuit for RF System-On-Chip Applications", Journal of the Korea Institute of Information and Communication Engineering, vol. 15, no.3, (2011), pp. 632-638.

[10] H. A. Shah, L. L. Hasan and I. S. Koo, "Optimized and Portable FPGA-Based Systolic Cell Architecture for Smith-Waterman-Based DNA Sequence Alignment", Journal of Information and Communication Convergence Engineering, vol. 14, no. 1, (2016), pp. 26-34.

[11] W.-Y. Uhm, K.-K. Ryu and S.-C. Kim, "Design of a 94-GHz Single Balanced Mixer Using Planar Schottky Diodes with a Nano-dot Structure on a GaAs Substrate", Journal of Information and Communication Convergence Engineering, vol. 14, no. 1, (2016), pp. 35-39.

[12] Y. S. Lin and J. N. Chang, "A 24-GHz power amplifier with Psat of $15.9 \mathrm{dBm}$ and PAE of $14.6 \%$ using standard $0.18 \mu \mathrm{m}$ CMOS technology", Analog Integrated Circuits and Signal Processing, vol. 79, no. 3, (2014), pp. 427-435.

[13] N.C. Kuo, J.C. Kao, C.C. Kuo, and H. Wang, "K-band CMOS power amplifier with adaptive bias for enhancement in back-off efficiency", IEEE MTT-S International Microwave Symposium Digest, vol. 11, no. 1, Baltimore, USA, (2011).

[14] A. Komijani, A. Natarajan, and A. Hajimiri, "A 24-GHz, +14.5-dBm Fully Integrated Power Amplifier in 0.18- $\mu \mathrm{m}$ CMOS", IEEE Journal of Solid-State Circuits, vol. 40, no. 9, (2005), pp. 1901-1908.

[15] J. Wenger, "Automotive Radar-Status and Perspectives", IEEE Compound Semiconductor Integrated Circuit Symposium, vol. 2, no. 1, (2005).

[16] T. S. D. Cheung, and J. R. Long, “A 21-26-GHz SiGe Bipolar Power Amplifier MMIC”, IEEE Journal of Solid-State Circuits, vol. 40, no. 12, (2005), pp. 2583-2597. 